ESAIM: PROCEEDINGS, March 2012, Vol. 35, p. 216-221

Fédération Denis Poisson (Orléans-Tours) et E. Trélat (UPMC), Editors

\title{
A MATRIX-BASED NUMERICAL METHOD FOR THE SIMULATION OF THE TWO-DIMENSIONAL SINE-GORDON EQUATION ${ }^{* * *}$
}

\author{
FRANCISCO DE LA HOZ ${ }^{1}$
}

\begin{abstract}
This paper describes a numerical method for the two-dimensional sine-Gordon equation over a rectangular domain using differentiation matrices, in the theoretical frame of matrix differential equations.

Résumé. Cette courte note décrit une méthode numérique pour l'équation de sine-Gordon bidimensionnelle sur un domaine rectangulaire en utilisant des matrices de différenciation, dans le cadre théorique des équations différentielles matricielles.
\end{abstract}

\section{INTRODUCTION}

In this paper, we consider the sine-Gordon equation (SGE):

$$
u_{t t}(\bar{x}, t)=\triangle u(\bar{x}, t)-\sin (u(\bar{x}, t)), \quad u: \mathbb{R}^{N} \times \mathbb{R}^{+} \rightarrow \mathbb{R} .
$$

This equation is particularly relevant, because it appears in many areas in mathematics, mechanics and theoretical physics. It describes, for instance, the deformation of a nonlinear crystal-lattice, dislocation in solids, properties of ferromagnets, etc. For many of its applications, see for example [19, pg. 1448], [12, pg. 199], or the introduction of [1], together with their references.

As with other equations, SGE has been most intensively studied for the one-dimensional case. Nevertheless, in the last years, there have appeared a pretty large number of papers devoted to studying numerically the two-dimensional SGE defined over a rectangular domain:

$$
u_{t t}(x, y, t)=u_{x x}+u_{y y}-\sin (u(x, y, t)), \quad(x, y) \in\left[-L_{x},+L_{x}\right] \times\left[-L_{y},+L_{y}\right],
$$

and imposing almost exclusively homogeneous Neumann boundary conditions ( [3], [4], [21, pg. 134], [11], [17], [18], [10], etc.). Despite using different techniques, the authors do not compare their methods; and their results for the test problems taken from [6] look very similar. On the other hand, in a recently submitted paper [9] (which can be obtained on request), F. de la Hoz and F. Vadillo have developed a pseudo-spectral matrix-based method to solve numerically SGE over an axiparallel rectangular domain in an arbitrary number of spatial dimensions, and with arbitrary time-dependent Neumann boundary conditions. The idea is to discretize the domain at the Chebyshev-Lobatto nodes, approximating the partial derivatives by means of differentiation

\footnotetext{
* This work was supported by MEC (Spain), with the project MTM2007-62186, and by the Basque Government, with the project IT-305-0\%.

** I want to thank Fernando Vadillo for his ongoing collaboration on this research area.

1 University of the Basque Country, Department of Applied Mathematics, Plaza de La Casilla 3, 48012 Bilbao (Spain); email: francisco.delahoz@ehu.es

(C) EDP Sciences, SMAI 2012
} 
matrices, and to use a fourth-order Runge-Kutta method with integrating factor [20, chap. 10] to advance in time, avoiding completely the calculation of matrix exponentials and of tensorization.

In this short paper, we announce the main results of [9] for the two dimensional case, i.e., we develop a matrixbased numerical method for the two-dimensional SGE over a rectangular domain with arbitrary Neumann boundary conditions. The structure of the paper is as follows: In Section 1, we formulate the matrix problem; in Section 2, we solve the corresponding linear problem; in Section 3, we develop a first-order numerical scheme with integrating factor; in Section 4, we perform the numerical tests; and, finally, in Section 5, we draw the main conclusions.

\section{Formulation of the MAtrix PROBlem}

Spectral methods have been successfully applied to time-dependent partial differential equations (PDE) and there is an ample literature on this subject (see for instance [14], [2] and [20], as well as the more classic references [15] and [5]). The idea is to approximate the solution $u(x, y, t)$ by a finite sum:

$$
u(x, y, t) \approx U(x, y, t)=\sum_{k=0}^{n_{x}} \sum_{l=0}^{n_{y}} a_{k l}(t) \phi_{k}\left(\frac{x}{L_{x}}\right) \phi_{l}\left(\frac{y}{L_{y}}\right)
$$

where $\phi_{m}(\cdot)=\cos (m \arccos (\cdot))$ is the Chebyshev polynomial of degree $m ; x \in\left[-L_{x}, L_{x}\right]$ and $y \in\left[-L_{y}, L_{y}\right]$. This approximation is an equality (i.e., a spectral equality) for a large enough number of addends, i.e., a large enough spectrum, except for errors smaller than the accuracy of the machine.

Chebyshev polynomials appear when dealing with non-periodic problems like (2), while, for periodic problems, trigonometric polynomials are the correct choice. There are different approaches to determine the expansion coefficients $a_{k l}(t)$ : we will focus on pseudo-spectral methods, where $a_{k l}(t)$ are required to make the residual equal zero at as many (suitably chosen) spatial points $\left(x_{j}, y_{i}\right)$ as possible. In this case, the natural choice are the Lobatto-Chebyshev nodes, i.e., $\left(x_{j}, y_{i}\right)=\left(L_{x} \cos \left(j \pi / n_{x}\right), L_{y} \cos \left(i \pi / n_{y}\right)\right), 0 \leq j \leq n_{x}, 0 \leq i \leq n_{y}$.

In our matrix-based philosophy, we do not obtain explicitly the coefficients $a_{k l}(t)$. Instead, our evolution variable is the matrix $\mathbf{U}(t) \in \mathcal{M}_{\left(n_{y}+1\right) \times\left(n_{x}+1\right)}$, where $U_{i j}(t) \equiv U\left(x_{j}, y_{i}, t\right), 0 \leq j \leq n_{x}, 0 \leq i \leq n_{y}$. Notice that we write $\left(x_{j}, y_{i}\right)$ rather than $\left(x_{i}, y_{j}\right)$, to be coherent with MatlaB commands, such as meshgrid. To discretize (2), we approximate $u_{x x}$ and $u_{y y}$ by means of the Chebyshev differentiation matrix $\mathbf{D}$ [20, chap. 6] [22] [13]:

$$
u_{y y}\left(x_{j}, y_{i}\right) \approx\left(\frac{1}{L_{y}^{2}} \mathbf{D}^{2}\right) \cdot \mathbf{U}, \quad u_{x x}\left(x_{j}, y_{i}\right) \approx \mathbf{U} \cdot\left(\frac{1}{L_{x}^{2}} \mathbf{D}^{2}\right)^{T}
$$

where $\mathbf{D}^{T}$ denotes the transpose of $\mathbf{D}$. Then, (2) becomes

$$
\mathbf{U}_{t t}(t)=\mathbf{U}(t) \cdot\left(\frac{1}{L_{x}^{2}} \mathbf{D}^{2}\right)^{T}+\left(\frac{1}{L_{y}^{2}} \mathbf{D}^{2}\right) \cdot \mathbf{U}(t)-\sin (\mathbf{U}(t))
$$

where the sine is applied pointwise. In the discretization of (2), only the inner elements $U_{i j}$ of the matrix are considered, i.e., those with $1 \leq j \leq n_{x}-1,1 \leq i \leq n_{y}-1$. Therefore, we have to recover the border elements $U_{0 j}, U_{n_{y} j}, U_{i 0}$ and $U_{i n_{x}}$ in function of the inner elements of $\mathbf{U}$ by means of the boundary conditions corresponding to (2). More precisely, if $\tilde{\mathbf{U}} \in \mathcal{M}_{\left(n_{y}-1\right) \times\left(n_{x}-1\right)}$ denotes the inner points of $\mathbf{U}$, then it is not difficult [7] to find matrices $\mathbf{P}_{1} \in \mathcal{M}_{\left(n_{y}+1\right) \times\left(n_{x}-1\right)}, \mathbf{P}_{2} \in \mathcal{M}_{\left(n_{y}-1\right) \times\left(n_{x}+1\right)}$ and $\mathbf{Q} \in \mathcal{M}_{\left(n_{y}+1\right) \times\left(n_{x}+1\right)}$, such that

$$
\mathbf{U}(t)=\mathbf{P}_{1} \cdot \tilde{\mathbf{U}}(t) \cdot \mathbf{P}_{2}+\mathbf{Q}(t)
$$

is a spectral equality. $\mathbf{P}_{1}$ and $\mathbf{P}_{2}$ are always time-independent, while $\mathbf{Q}$ is time-independent only if the boundary conditions are time-independent, being zero if we are dealing with homogeneous boundary conditions. 
Introducing (5) into (4) and restricting ourselves to the inner points, (4) becomes

$$
\mathbf{U}_{t t}(t)=\mathbf{A} \cdot \mathbf{U}(t)+\mathbf{U}(t) \cdot \mathbf{B}^{T}+\mathbf{C}(t)-\sin (\mathbf{U}(t))
$$

where, in order not to burden the notation, and without lost of generality, we have omitted the tildes of $\tilde{\mathbf{U}}$. C is time-independent if the boundary conditions are time-independent, and zero if the boundary conditions are homogeneous. A and $\mathbf{B}$ are actually the second-order Chebyshev differentiating matrices with homogeneous Neumann boundary-conditions and applied to the inner nodes; if $n_{x}=n_{y}$, then $L_{y}^{2} \mathbf{A}=L_{x}^{2} \mathbf{B}$. We will solve numerically (6) and, once calculated its evolution, will recover the border elements of $\mathbf{U}(t)$ from $(5)$.
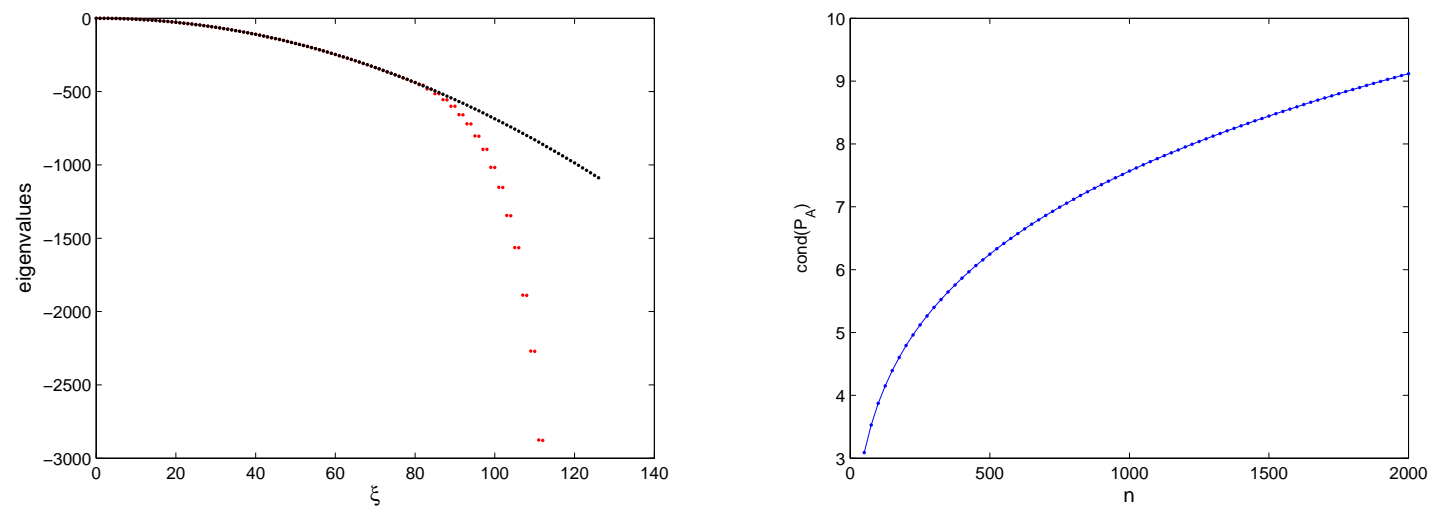

Figure 1. Left: Eigenvalues $\lambda_{\xi}(\mathbf{A})$ of the second-order differentiation matrix $\mathbf{A}$ with homogeneous Neumann boundary conditions, $n_{y}=128, L_{y}=6$ (red), versus the corresponding eigenvalues $\lambda_{\xi}=-\left(\xi \pi / 2 L_{y}\right)^{2}$ of the continuous problem $u_{x x}=\lambda u, u_{x}\left(-L_{y}\right)=u_{x}\left(+L_{y}\right)=0$ (black). Right: Condition number of $\mathbf{P}_{A}$, as a function of the number of spatial nodes $n_{y}$.

At this point, two crucial observations should be done. The first one is that all the eigenvalues of $\mathbf{A}$ and $\mathbf{B}$ are negative, except for one, which equals zero except maybe for infinitesimal rounding errors. This fact, which is required for stability, is evident from a numerical point of view from the left hand-side of Figure 1 . The second crucial observation is the well-conditionedness of the matrices of eigenvectors $\mathbf{P}_{A}$ and $\mathbf{P}_{B}$ that appear in the diagonal decomposition of $\mathbf{A}$ and $\mathbf{B}$, i.e., $\mathbf{D}_{A}=\mathbf{P}_{A}^{-1} \cdot \mathbf{A} \cdot \mathbf{P}_{A}$ and $\mathbf{D}_{B}=\mathbf{P}_{B}^{-1} \cdot \mathbf{B} \cdot \mathbf{P}_{B} ;$ this is required to avoid direct calculation of exponential matrices, which is a central idea of this paper. In the right-hand side of Figure 1, we have plotted the 2-norm condition number of the eigenvector matrix $\mathbf{P}_{A}$ of $\mathbf{A} \in \mathcal{M}_{\left(n_{y}-1\right) \times\left(n_{y}-1\right)}$, for $L=6$, and different $n_{y}$, being evident that the condition numbers are small and grow sublinearly.

\section{Solving the Linear Problem}

The integrating-factor method is based on the idea that a problem with a linear part plus a nonlinear one can be transformed, so that its linear part is solved exactly (see [20, chap. 10], [8], and their references). Hence, to apply an integrating factor to (6), we need to solve first its linear part

$$
\mathbf{U}_{t t}=\mathbf{A} \cdot \mathbf{U}+\mathbf{U} \cdot \mathbf{B}^{T}
$$

with initial data $\mathbf{U}(0), \mathbf{U}_{t}(0)$. Denoting $\mathbf{V}(t) \equiv \mathbf{U}_{t}(t),(7)$ can be solved efficiently by the following theorem $[9]$ : 
Theorem 2.1. Given the time-independent matrices $\mathbf{A} \in \mathcal{M}_{\left(n_{y}-1\right) \times\left(n_{y}-1\right)}$, and $\mathbf{B} \in \mathcal{M}_{\left(n_{x}-1\right) \times\left(n_{x}-1\right)}$, with diagonal decompositions $\mathbf{D}_{A}=\mathbf{P}_{A}^{-1} \cdot \mathbf{A} \cdot \mathbf{P}_{A}$ and $\mathbf{D}_{B}=\mathbf{P}_{B}^{-1} \cdot \mathbf{B} \cdot \mathbf{P}_{B}$, the solution of (7) is

$$
\begin{aligned}
\mathbf{U}(t)= & \mathbf{P}_{A} \cdot\left[\left(\mathbf{P}_{A}^{-1} \cdot \mathbf{U}(0) \cdot\left(\mathbf{P}_{B}^{-1}\right)^{T}\right) \circ \cos \left(t \sqrt{-\Lambda_{A B}}\right)\right] \cdot \mathbf{P}_{B}^{T} \\
& +\mathbf{P}_{A} \cdot\left[\left(\mathbf{P}_{A}^{-1} \cdot \mathbf{U}_{t}(0) \cdot\left(\mathbf{P}_{B}^{-1}\right)^{T}\right) \circ\left(\sqrt{-\Lambda_{A B}}\right)^{-1} \circ \sin \left(t \sqrt{-\Lambda_{A B}}\right)\right] \cdot \mathbf{P}_{B}^{T} \\
\mathbf{V}(t)= & \mathbf{P}_{A} \cdot\left[-\left(\mathbf{P}_{A}^{-1} \cdot \mathbf{U}(0) \cdot\left(\mathbf{P}_{B}^{-1}\right)^{T}\right) \circ \sqrt{-\Lambda_{A B}} \circ \sin \left(t \sqrt{-\Lambda_{A B}}\right)\right] \cdot \mathbf{P}_{B}^{T} \\
& +\mathbf{P}_{A} \cdot\left[\left(\mathbf{P}_{A}^{-1} \cdot \mathbf{U}_{t}(0) \cdot\left(\mathbf{P}_{B}^{-1}\right)^{T}\right) \circ \cos \left(t \sqrt{-\Lambda_{A B}}\right)\right] \cdot \mathbf{P}_{B}^{T},
\end{aligned}
$$

where all the operations are pointwise; $\circ$ denotes the Hadamard or pointwise product of two matrices [16]; and $\boldsymbol{\Lambda}_{A B}=\left[\lambda_{i j}\right]$ is the matrix whose elements are $\lambda_{i j}=\lambda_{i}\left(\mathbf{D}_{A}\right)+\lambda_{j}\left(\mathbf{D}_{B}\right)$, for $i=1, \ldots, n_{y}-1, j=1, \ldots, n_{x}-1$.

Observe that if $\lambda_{i j}=0$, we take $\left(\sqrt{-\lambda_{i j}}\right)^{-1} \sin \left(t \sqrt{-\lambda_{i j}}\right) \equiv t$, so zero eigenvalues cause no concern. Moreover, since we have proven numerically (left-hand side of Figure 1) that $\lambda_{i j} \leq 0, \mathbf{U}(t)$ and $\mathbf{V}(t)$ are real and bounded $\forall t>0$. On the other hand, if there were $\lambda_{i j}>0$, there would be stability issues as $t \rightarrow \infty$.

\section{INTEGRATING FACTOR WITH FORWARD EULER DISCRETIZATION}

Let us transform (6) by means of the vec operator [16]:

$$
\left(\begin{array}{c}
\operatorname{vec}(\mathbf{U}) \\
\operatorname{vec}(\mathbf{V})
\end{array}\right)_{t}=\mathbf{M} \cdot\left(\begin{array}{c}
\operatorname{vec}(\mathbf{U}) \\
\operatorname{vec}(\mathbf{V})
\end{array}\right)+\left(\begin{array}{c}
0 \\
\operatorname{vec}(\mathbf{C})-\sin (\operatorname{vec}(\mathbf{U}))
\end{array}\right)
$$

where $\mathbf{V}=\mathbf{U}_{t}$, and $\mathbf{M}$ is the block matrix

$$
\mathbf{M}=\left(\begin{array}{cc}
0 & \mathbf{I} \\
\mathbf{B} \oplus \mathbf{A} & 0
\end{array}\right)
$$

where $\oplus$ denotes the Kronecker sum [16], and we have used that $\operatorname{vec}\left(\mathbf{A} \cdot \mathbf{U}(t)+\mathbf{U}(t) \cdot \mathbf{B}^{T}\right) \equiv(\mathbf{B} \oplus \mathbf{A}) \cdot \operatorname{vec}(\mathbf{U})$. In (9), there is a linear part plus a non-linear part; to get rid of the linear part, we multiply at both sides by the integrating factor $\exp (-t \mathbf{M})$, where exp denotes the matrix exponential, getting

$$
\left(\exp (-t \mathbf{M}) \cdot\left(\begin{array}{c}
\operatorname{vec}(\mathbf{U}) \\
\operatorname{vec}(\mathbf{V})
\end{array}\right)\right)_{t}=\exp (-t \mathbf{M}) \cdot\left(\begin{array}{c}
0 \\
\operatorname{vec}(\mathbf{C})-\sin (\operatorname{vec}(\mathbf{U}))
\end{array}\right)
$$

Using a forward Euler discretization in time, it becomes

$$
\exp \left(-t^{n+1} \mathbf{M}\right) \cdot\left(\begin{array}{c}
\operatorname{vec}\left(\mathbf{U}^{n+1}\right) \\
\operatorname{vec}\left(\mathbf{V}^{n+1}\right)
\end{array}\right)-\exp \left(-t^{n} \mathbf{M}\right) \cdot\left(\begin{array}{c}
\operatorname{vec}\left(\mathbf{U}^{n}\right) \\
\operatorname{vec}\left(\mathbf{V}^{n}\right)
\end{array}\right)=\Delta t \exp \left(-t^{n} \mathbf{M}\right) \cdot\left(\begin{array}{c}
0 \\
\operatorname{vec}\left(\mathbf{C}^{n}\right)-\sin \left(\operatorname{vec}\left(\mathbf{U}^{n}\right)\right)
\end{array}\right)
$$

Multiplying at both sides by $\exp \left(t^{n+1} \mathbf{M}\right)$, we get finally

$$
\left(\begin{array}{c}
\operatorname{vec}\left(\mathbf{U}^{n+1}\right) \\
\operatorname{vec}\left(\mathbf{V}^{n+1}\right)
\end{array}\right)=\exp (\Delta t \mathbf{M}) \cdot\left[\left(\begin{array}{c}
\operatorname{vec}\left(\mathbf{U}^{n}\right) \\
\operatorname{vec}\left(\mathbf{V}^{n}\right)
\end{array}\right)+\Delta t\left(\begin{array}{c}
0 \\
\operatorname{vec}\left(\mathbf{C}^{n}\right)-\sin \left(\operatorname{vec}\left(\mathbf{U}^{n}\right)\right)
\end{array}\right)\right]
$$


which is obviously the solution of (7) at time $t=\Delta t$, with initial data $\mathbf{U}(0)=\mathbf{U}^{n}$, and $\mathbf{V}(0)=\mathbf{V}^{n}+$ $\Delta t\left(\operatorname{vec}\left(\mathbf{C}^{n}\right)-\sin \left(\operatorname{vec}\left(\mathbf{U}^{n}\right)\right)\right)$; hence, by Theorem 2.1,

$$
\begin{aligned}
\mathbf{U}^{n+1}= & \mathbf{P}_{A} \cdot\left[\left(\mathbf{P}_{A}^{-1} \cdot \mathbf{U}^{n} \cdot\left(\mathbf{P}_{B}^{-1}\right)^{T}\right) \circ \cos \left(\Delta t \sqrt{-\Lambda_{A B}}\right)\right. \\
& \left.+\left(\mathbf{P}_{A}^{-1} \cdot\left[\mathbf{V}^{n}+\Delta t\left(\mathbf{C}^{n}-\sin \left(\mathbf{U}^{n}\right)\right)\right] \cdot\left(\mathbf{P}_{B}^{-1}\right)^{T}\right) \circ\left(\sqrt{-\Lambda_{A B}}\right)^{-1} \circ \sin \left(\Delta t \sqrt{-\Lambda_{A B}}\right)\right] \cdot \mathbf{P}_{B}^{T} \\
\mathbf{V}^{n+1}= & \mathbf{P}_{A} \cdot\left[-\left(\mathbf{P}_{A}^{-1} \cdot\left[\mathbf{V}^{n}+\Delta t\left(\mathbf{C}^{n}-\sin \left(\mathbf{U}^{n}\right)\right)\right] \cdot\left(\mathbf{P}_{B}^{-1}\right)^{T}\right) \circ \sqrt{-\Lambda_{A B}} \circ \sin \left(\Delta t \sqrt{-\Lambda_{A B}}\right)\right. \\
& \left.+\left(\mathbf{P}_{A}^{-1} \cdot \mathbf{U}^{n} \cdot\left(\mathbf{P}_{B}^{-1}\right)^{T}\right) \circ \cos \left(\Delta t \sqrt{-\Lambda_{A B}}\right)\right] \cdot \mathbf{P}_{B}^{T}
\end{aligned}
$$

In brief, we have tensorized (6), applied the integrating factor, discretized the equation in time, and destensorized the resulting scheme. The previous ideas can be easily extended to higher-order schemes, by applying a higherorder discretization to (11). In the following section, for comparison's sake, we have also considered a fourthorder Runge-Kutta discretization of (11).
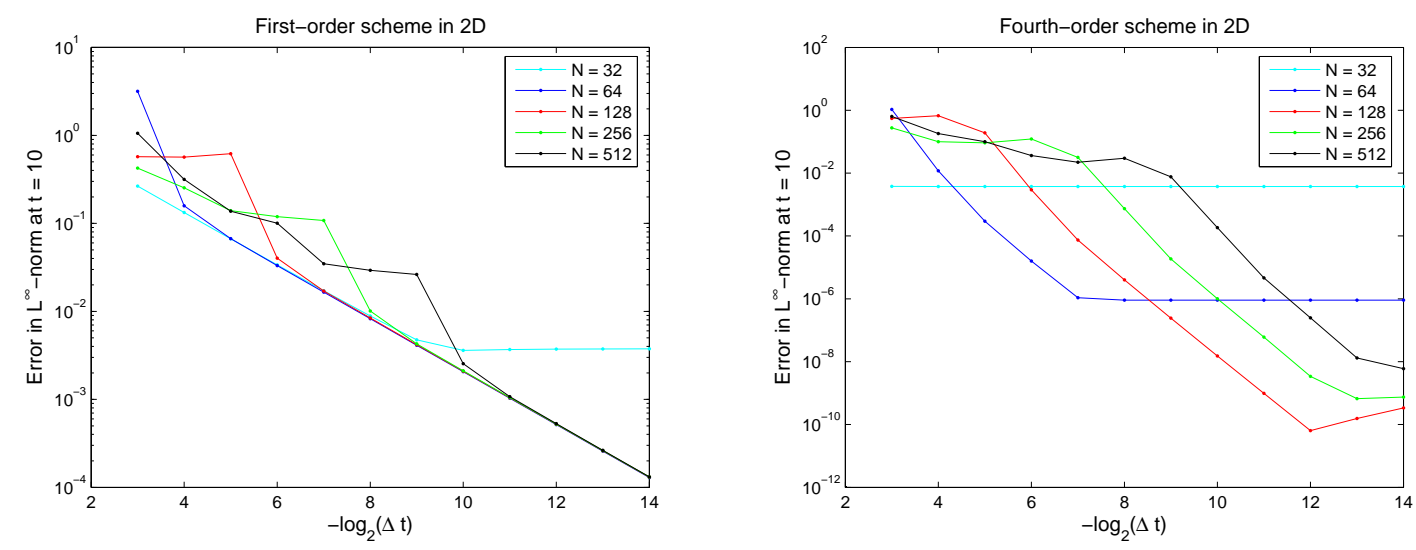

FiguRE 2. Errors in $L^{\infty}$-norm at $t=10$, for $L_{x}=L_{y}=6$, and different $n_{x}=n_{y}=N$ and $\Delta t$, corresponding to the first-order scheme (left), and to the fourth-order scheme (right).

\section{NumERICAL TESTS}

We have considered the theoretical solution of $(2), u(x, y, t)=4 \arctan (\exp [(\sqrt{2} / 2) x+(\sqrt{6} / 2) y-t]),(x, y) \in$ $[-6,6]^{2}, \forall t \geq 0$; introducing exactly the initial data $u(x, y, 0)$ and $u_{t}(x, y, 0)$, and the inhomogeneous Neumann boundary conditions $\partial_{x} u( \pm 6, y, t)$, and $\partial_{y} u(x, \pm 6, t), \forall t$. We have executed the first-order scheme described above, as well as a fourth-order Runge-Kutta with integrating factor [9], for $L_{x}=L_{y}=6$ and different $n_{x}=n_{y}$. Figure 2 shows the errors obtained in $L^{\infty}$-norm at $t=10$, for different $\Delta t$; notice that $\max _{(x, y)} u(x, y, 10) \approx 5.48$. The left-hand side and right-hand side correspond respectively to the first and fourth-order schemes; the orders are evident from the slopes of the (approximately) straight lines that appear: while the first-order may need a prohibitive $\Delta t$ to yield good results, the fourth-order method offers a highly remarkable accuracy.

To obtain the highest possible accuracy, a minimum number of nodes $N$ is required. For example, with $N=32$ and $N=64$, the minimum possible errors are only about $3.7581 \cdot 10^{-3}$ and $9.0774 \cdot 10^{-7}$, respectively. On the other hand, the method is very stable, even for big $N$.

From the right-hand side, it is apparent that if we double $N$, we have to divide $\Delta t$ by four to get a similar accuracy. Therefore, we have a $\Delta t=\mathcal{O}\left(1 / N^{2}\right)$ restriction if we want to get the maximum accuracy. Although 
the results get slightly worse when we increase $N$, they are still very good, even for large $N$. For instance, for $N=512$ and $\Delta t=2^{-14}$, we have an absolute error of about $6.0143 \cdot 10^{-9}$.

\section{Conclusions}

We have developed a new numerical matrix-based method with integrating factor to solve efficiently and accurately the two-dimensional SGE (2), avoiding the explicit calculation of matrix exponentials and the use of Kronecker tensor products. To understand why avoiding tensor products is vital, let be $\mathbf{A}, \mathbf{B} \in \mathcal{M}_{N \times N}$, where all the components of both $\mathbf{A}$ and $\mathbf{B}$ are positive (so that there are no cancellations); then it can be shown that $\mathbf{B} \oplus \mathbf{A} \in \mathcal{M}_{N^{2} \times N^{2}}$ has exactly $2 N^{3}-N^{2}$ non-zero elements, i.e. a sparsity ratio of $\mathcal{O}(1 / N)$. Therefore, if $N=511, \mathbf{A}$ and $\mathbf{B}$ have just 261121 elements, versus $\mathbf{B} \oplus \mathbf{A}$, which has 68184176641 elements, of which 266604541 are nonzero! In other words, the problem becomes quickly intractable. Another virtue of the non-tensor approach is that it can be extended to higher dimensions [9], overcoming the curse of dimensionality.

The method can be applied, with little modification, to other types of nonlinear Klein-Gordon equations, with other types of boundary conditions. Furthermore, the techniques of this paper are not necessarily restricted to axiparallel rectangular domains. Indeed, as long as the spatially semi-discretized problem can be written in the form of (6), with eventually another nonlinear term, the general ideas in this paper are applicable.

\section{REFERENCES}

1. E. L. Aero, A. N. Bulygin, and Y. V. Pavlov, Solutions of the three-dimensional sine-Gordon equation, Theoretical and Mathematical Physics 158 (2009), 313-319.

2. J. P. Boyd, Chebyshev and Fourier Spectral Methods, Dover, 2001.

3. A. G. Bratsos, The solution of the two-dimensional sine-Gordon equation using the method of lines, J. Comput. Appl. Math. 206 (2007), 251-277.

4. _ An improved numerical scheme for the sine-Gordon equation in 2+1 dimensions, Int. J. Numer. Meth. Engng $\mathbf{7 5}$ (2008), 787-799.

5. C. Canuto, M. Y. Hussain, A. Quarteroni, and T. A. Zang, Spectral Methods in Fluid Dynamics, Springer, 1988.

6. P. L. Christiansen and P. S. Lomdahl, Numerical solutions of $2+1$ dimensional sine-Gordon solitons, Physica 2D (1981), 482-494.

7. F. de la Hoz and F. Vadillo, A Simple Method for Two-Dimensional Advection-Diffusion Equations via Operational Matrices, preprint.

8. _ An integrating factor for nonlinear Dirac equations, Computer Physics Communications 181 (2010), 1195-1203.

9. - Numerical simulation of the $N$-dimensional sine-Gordon equation via operational matrices, Computer Physics Communications 183 (2012), 864-879.

10. M. Dehghan and A. Ghesmati, Numerical simulation of two-dimensional sine-Gordon solitons via a local weak meshless technique based on the radial point interpolation method (RPIM), Computer Physics Communications 181 (2010), 772-786.

11. M. Dehghan and A. Shokri, A numerical method for solution of the two-dimensional sine-Gordon equation using the radial basis functions, Mathematics and Computers in Simulation 79 (2008), 700-715.

12. P. G. Drazin and R. S. Johnson, Solitons : an introduction, Cambridge University Press, 1989.

13. E. M. E. Elbarbary and S. M. El-Sayed, Higher order pseudospectral differentiation matrices, Applied Numerical Mathematics 55 (2005), no. 4, 425-438.

14. B. Fornberg, A Practical Guide to Pseudospectral Methods, Cambridge University Press, 1998.

15. D. Gottlieb and S. A. Orszag, Numerical Analysis of Spectral Methods: Theory and Applications, SIAM, 1977.

16. N. J. Higham, Function of Matrices. Theory and Computation, SIAM, 2008.

17. D. Mirzaei and M. Dehghan, Boundary element solution of the two-dimensional sine-Gordon equation using continuous linear elements, Engineering Analysis with Boundary Elements 33 (2009), 12-24.

18. _ Meshless local Petrov-Galerkin (MLPG) approximation to the two dimensional sine-Gordon equation, J. Comput. Appl. Math. 233 (2010), 2737-2754.

19. A. C. Scott, F. Y. F. Chu, and D. W. McLaughlin, The Soliton: A New Concept in Applied Science, Proceedings of the IEEE 61 (1973), no. 10, 1443-1483.

20. L. N. Trefethen, Spectral Methods in MATLAB, SIAM, 2000.

21. E. H. Twizell, Computational Methods for Partial Differential Equations, John Wiley \& Sons, 1984.

22. J. A. C. Weideman and S. C. Reddy, A MATLAB Differentiation Matrix Suite, ACM Trans. Math. Software 26 (2000), no. 4, $465-519$. 